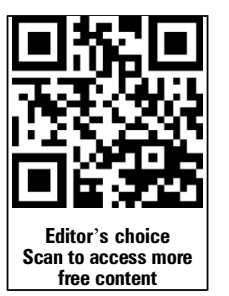

${ }^{1}$ Research Department, ClearWay Minnesota, Minneapolis, Minnesota, USA ${ }^{2}$ Department of Oncology, Georgetown University, Lombardi Comprehensive Cancer Centre, Washington DC, USA

\section{Correspondence to}

Dr Michael S Amato, Research Department, ClearWay

Minnesota Minneapolis, MN 55425, USA:

mamato@clearwaymn.org

Received 16 January 2015 Accepted 21 April 2015 Published Online First 17 June 2015

\title{
How to define e-cigarette prevalence? Finding clues in the use frequency distribution
}

\author{
Michael S Amato, ${ }^{1}$ Raymond G Boyle, ${ }^{1}$ David Levy ${ }^{2}$
}

\begin{abstract}
Objective E-cigarette use has rapidly increased. Recent studies define prevalence using a variety of measures; competing definitions challenge cross-study comparison. We sought to understand patterns of use by investigating the number of days out of the past 30 days when adults had used e-cigarettes.
\end{abstract}

Design We used the 2014 Minnesota Adult Tobacco Survey, a random digit dial population survey ( $n=9304$ adults). Questions included ever using e-cigarettes, number of days used in the past 30 days and reasons for use. Smoking status was determined by combustible cigarette use. Histograms of e-cigarette use were visually inspected for current, former and never smokers with any 30-day e-cigarette use. Different definitions of current use were compared.

Results Use $\leq 5$ days in the past 30 days demarcated a cluster of infrequent users at the low end of the distribution. Among those with use in the past 30 days, infrequent users were the majorities of current $(59 \%)$ and never smokers $(89.5 \%)$, but fewer than half of former smokers $(43.2 \%)$. Infrequent users were more likely to cite curiosity and less likely to cite quitting/ cutting down other tobacco use as reasons for use. Conclusions Defining adult prevalence as any use in the past 30 days may include experimenters unlikely to continue use, and is of questionable utility for population surveillance of public health trends over time. Defining prevalence as $>5$ days excludes those infrequent users.

\section{INTRODUCTION}

The use of electronic cigarettes (e-cigarettes) has increased rapidly in the past several years. ${ }^{1-6}$ Sales in 2014 are estimated to be nearly \$2 billion and one recent business analysis predicted they could ultimately surpass combusted cigarette sales. ${ }^{2} 7$ Although helpful, sales data provide no information about how individuals use the product. For ecigarette users, surveillance or other surveys are necessary. The extent of an individual's e-cigarette use may be an important factor related to transition of smokers away from cigarette use (ie, cessation), as well as potential initiation of non-smokers and relapse of former smokers into cigarette use. Understanding the population effects of e-cigarette use on these transitions will be crucial in determining definitions of use that are most useful in gauging the public health impact of that use. In particular, it will be important to distinguish more established patterns of use from short-term (eg, experimental use) in gauging the effects of ecigarettes on population health, since relative risks are likely to be dependent on long-term use.
One of the challenges for survey research is the importance of asking questions to reliably measure meaningful use of a tobacco product. A 2009 review of major tobacco surveillance surveys identified inconsistent methodology as an area for improvement, particularly with regard to tobacco products other than cigarettes. $^{8}$ A standard measure of adult cigarette smoking, based on a lifetime minimum threshold of 100 cigarettes smoked and reporting currently smoking 'every day or some days', is widely used and allows straightforward comparison across studies. The measure's wide adoption has been attributed in part to its efficiency for reducing the survey burden on both researchers and respondents; however, it has also faced criticism for being arbitrarily chosen and inadequately sensitive, particularly, but not exclusively for youth samples. ${ }^{9}$ In light of these concerns, the 2014 US Surgeon General's report on smoking estimated current cigarette smoking prevalence for youth and young adults based on having smoked all or part of at least one cigarette in the past 30 days. ${ }^{10}$ Nonetheless, surveys such as the National Survey on Drug Use and Health, which have adopted the 30-day measure, have also retained the historically more common measure in recognition of the importance of maintaining comparability with previous studies that used the historically more common measure.

In contrast, a historically common definition of e-cigarette current use prevalence does not exist; a variety of definitions can be found in the literature. Reflecting the shift in measurement of cigarette smoking prevalence, many recent studies have adopted a measure of adult e-cigarette current use based on any use in the past 30 days. ${ }^{5} 11-13$ Alternatively, some researchers have adopted language from the conventional cigarette current use definition of use on every day or some days, but without the lifetime threshold question. ${ }^{4}{ }^{6}$ 14-16 Other studies have reported current use from questions that included multiple choice options for selfreported frequency. ${ }^{17-19}$

In addition to reporting prevalence of current use, several studies have investigated the distribution of use frequency by also reporting the prevalence of daily use, ${ }^{4} 1113$ or some other measure designed to exclude non-established users; however, their operationalisations of 'daily use' and 'established use' were not uniform. ${ }^{4}{ }^{11} \quad 14 \quad 20$ Excluding non-established users is important in some instances because evidence suggests they differ from established users not only in extent of participation, but also in their reasons for initially trying e-cigarettes. Specifically, individuals from a US sample who had ever used e-cigarettes were 
roughly twice as likely to continue their e-cigarette use if they had tried them for a goal-oriented reason, such as quitting or cutting down other tobacco use or using them in places where smoking was not allowed, compared to individuals who had tried e-cigarettes for a non-goal reason such as curiosity. ${ }^{16}$ The authors of that study argued that measures of current use which distinguish types of users in terms of their goals for e-cigarette use would be most useful to public health researchers and practitioners.

More broadly, competing definitions of prevalence based on different frequencies of use present a challenge to cross-study comparison. It is not immediately clear which definition provides the most useful information about how the introduction of e-cigarettes is or is not changing the overall environment of tobacco use, especially combusted cigarettes. The tobacco control community has been confronted with similar challenges of measurement with regards to changing patterns of use among young adults ${ }^{21}$ and less-than-daily cigarette smoking. ${ }^{22}$ While it is likely that no single question or set of questions will be able to fully satisfy all research needs, particularly with regard to the psychological mechanisms of behaviour change, large scale population surveillance efforts for public health may benefit from increased consistency in definitions of e-cigarette current use prevalence across studies. ${ }^{9}$

We had an opportunity to examine frequency of e-cigarette use, measured as a continuous variable, in a recent populationbased study. We focused on the shapes of the e-cigarette use frequency distributions among current smokers, former smokers and never smokers. To keep the study limited in scope, we restricted our definition of 'smoker' to use of cigarettes only; we did not investigate other forms of combustible tobacco. By examining the shapes of the distributions, we hoped to take advantage of information that is lost when focusing on measures of central tendency such as the mean or median. We then investigated the relationship between those distributions and respondents' reasons for using e-cigarettes. Our goal was to conduct a data-driven investigation in order to contribute evidence towards an eventual consensus regarding a standard definition of e-cigarette current use prevalence for the purpose of population surveillance.

\section{METHODS}

Data were collected as part of the 2014 Minnesota Adult Tobacco Survey, which uses a random digit dialing (RDD) methodology to obtain a cross-sectional sample of Minnesotan adults aged 18 years or older. Two sampling frames were used, one that included landline numbers and another that included cell phone numbers. Prescreening calls identified households and selected individuals within households; the main survey instrument was subsequently administered. A rigorous calling protocol was used, and letters were mailed to refusers and nonresponders when addresses were available. Attempts were made to convert refusers. RDD response rates calculated by American Association for Public Opinion Research methodology were $25.2 \%$ for the landline sampling frame and $18.2 \%$ for the cell phone frame. ${ }^{23}$ Sampling weights were calculated based on sampling frame response rates and demographic characteristics known to be correlated with tobacco use behaviours, to obtain unbiased population level estimates. More methodological detail is available at http://www.mnadulttobaccosurvey.org. The final sample in 2014 included 9304 participants; 9301 of the participants provided valid responses for the items considered in this analysis.
Smoking status was established according to the historically common Behavioural Risk Factor Surveillance System (BRFSS) methodology. Current smokers had smoked $\geq 100$ cigarettes in their lifetime and now smoked 'every day' or 'some days'; former smokers had smoked $\geq 100$ cigarettes in their lifetime, but now smoked 'not at all'; and never smokers had not smoked $\geq 100$ cigarettes in their lifetime. E-cigarette use was measured by two items. Participants were first asked, "Have you ever used an electronic cigarette, even just one time in your entire life?" Affirmative answers were followed by the question, "During the past 30 days, on how many days did you use e-cigarettes?" Responses were entered as integers by the data collector; respondents offering non-integer responses were prompted to provide an integer. Respondents who had ever used e-cigarettes were asked whether each of the following was a reason for use: to quit other tobacco products; to cut down on other tobacco products because they are affordable; because they are available in menthol flavour; because they are available in flavours other than menthol; to use them in places where other tobacco products are not allowed; curiosity about e-cigarettes; and because you believe these might be less harmful than other tobacco products. Based on the findings of Pepper $e a^{a l}{ }^{16}$, reasons were classified as goal oriented or non-goal oriented.

All analyses were conducted with the $\mathrm{R}$ software package, V.3.1.1, using the survey package V.3.30-3. All population estimates are presented with $95 \%$ CIs. Where direct comparisons of CIs are insufficient to establish significance at the $\alpha=0.05$ level (ie, where CIs overlapped), we report $\mathrm{p}$ values for pairwise comparisons that were calculated using linear regression.

\section{RESULTS}

In 2014, $17.7 \%$ (16.6\% to $18.8 \%)$ of Minnesota adults had tried e-cigarettes. Most current smokers $(70 \% \quad 66.7 \%$ to 73.4\%)) had tried e-cigarettes at least once in their life, compared to smaller percentages of former smokers $(15.9 \%$ (14\% to $17.9 \%)$ ) and never smokers (5.6\% (4.7\% to $6.5 \%)$; table 1$)$. Across all cigarette smoking statuses, fewer than half of those who had ever tried e-cigarettes in their lifetime reported having used them in the past 30 days: current smokers $38.9 \%(34.4 \%$ to $43.5 \%$ ); former smokers $30.3 \%$ (23.9\% to $36.7 \%$ ); never smokers $21.5 \%$ (15.3\% to $27.7 \%)$.

Histograms showing frequency of e-cigarette use for all individuals reporting any use in the past 30 days, separated by smoking status, are presented in figure 1. Based on the histograms, use less than or equal to 5 days in the past 30 days appeared to be a meaningful cut-off point to demarcate a cluster of respondents at the low end of each distribution whom we subsequently refer to as 'infrequent' users. Respondents who reported using an e-cigarette on 30 out of the past 30 days were categorised as 'daily' users. Respondents reporting use between 6 and 29 days (inclusive) were categorised as 'intermediate' users. Respondents who had used e-cigarettes in their lifetime, but not within the past 30 days, were categorised as 'past users'.

Current smokers were more likely to use e-cigarettes than former smokers or never smokers, across all frequency categories (table 1). Among current smokers who had used an ecigarette in the past 30 days, the most common category was infrequent user $(59 \%(51.5 \%$ to $66.5 \%))$, followed by intermediate users $(28.7 \%(21.8 \%$ to $35.6 \%))$ and daily users $(12.3 \%(7.2 \%$ to $17.4 \%))$.

In contrast with current smokers, the proportions of former smokers reporting e-cigarette use in the past 30 days who were categorised as infrequent users $(43.2 \%(30.2 \%$ to $56.1 \%))$ versus daily users $(40.9 \%(28.7 \%$ to $53 \%))$ did not statistically 
Table 1 Percentage of Minnesota adults using e-cigarettes, by increasingly restrictive definitions of prevalence based on reported frequency of use

\begin{tabular}{lllll}
\hline & $\begin{array}{l}\text { Ever in lifetime, } \\
\%(95 \% \mathrm{Cl})\end{array}$ & $\begin{array}{l}\text { At least } \mathbf{1} \text { day in past } 30, \\
\%(95 \% \mathrm{Cl})\end{array}$ & $\begin{array}{l}\text { More than } \mathbf{5} \text { days in past 30, } \\
\%(95 \% \mathrm{Cl})\end{array}$ & $\begin{array}{l}\text { Every day in past 30, } \\
\%(95 \% \mathrm{Cl})\end{array}$ \\
\hline $\begin{array}{l}\text { Overall } \\
\text { Cigarette smoking status }\end{array}$ & $17.7(16.6$ to 18.8$)$ & $6.0(5.2$ to 6.6$)$ & $2.4(2.0$ to 2.9$)$ & $1.1(0.8$ to 1.4$)$ \\
$\quad$ Current smoker $(14.4 \%$ of population) & $70.0(67.0$ to 73.4$)$ & $27.3(23.8$ to 30.7$)$ & $11.2(8.7$ to 13.7$)$ & $3.4(1.9$ to 4.8$)$ \\
$\quad$ Former smoker $(27.8 \%$ of population) & $15.9(14.0$ to 17.9$)$ & $4.8(3.6$ to 6.0$)$ & $2.7(1.9$ to 3.6$)$ & $2.0(1.3$ to 2.7$)$ \\
$\quad$ Never smoker $(57.8 \%$ of population) & $5.6(4.7$ to 6.5$)$ & $1.2(0.8$ to 1.6$)$ & $0.1(0.0$ to 0.2$)$ & $0.1(0.0$ to 0.1$)$ \\
\hline
\end{tabular}

differ $(\mathrm{p}=0.8)$. Comparatively fewer former smokers were categorised as intermediate users (16\% (6.1\% to $25.8 \%))$.

The majority of never smokers who reported some use of e-cigarettes in the past 30 days were infrequent users $(89.5 \%$ $(81.5 \%$ to $97.4 \%))$. Smaller proportions reported intermediate $(5.4 \%(0.4 \%$ to $10.5 \%))$ or daily $(5.1 \%(0.0 \%$ to $11.4 \%))$ e-cigarette use.

The reasons respondents cited for current or past use of e-cigarettes are presented in table 2. Significantly fewer infrequent users endorsed the goal-oriented reasons for using ecigarettes compared to daily users, $\mathrm{p}<0.05$ for all goal-oriented reasons. The proportions of infrequent users endorsing the goal-oriented reasons of quitting other tobacco products, cutting down on other tobacco products and affordability were all similarly significantly lower than the corresponding proportions of intermediate users. In contrast, a greater proportion of infrequent users cited curiosity as a reason for their e-cigarette use. The proportions of current, former and never smokers
Figure 1 Histograms of number of days respondents reported using an e-cigarette, for respondents with some 30-day use.
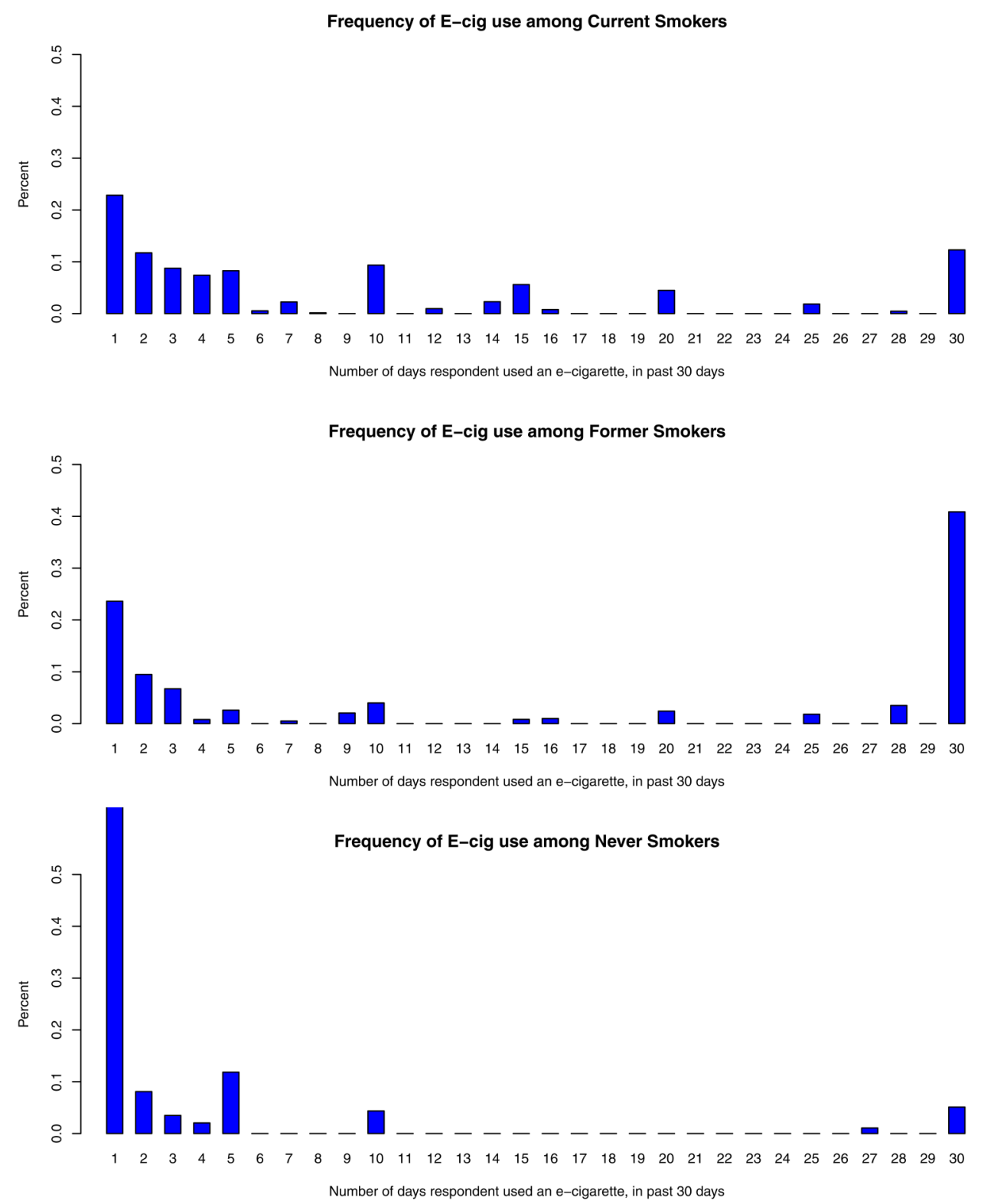

Number of days respondent used an e-cigarette, in past 30 days 
Table 2 Reasons cited by all adults who ever used e-cigarettes for why they use/have used them

\begin{tabular}{|c|c|c|c|c|}
\hline & $\begin{array}{l}\text { Past users } \\
0 \text { days in } \\
\text { past } 30 \\
\%(95 \% \mathrm{Cl})\end{array}$ & $\begin{array}{l}\text { Infrequent users } \\
1-5 \text { days in } \\
\text { past } 30 \\
\%(95 \% \mathrm{Cl})\end{array}$ & $\begin{array}{l}\text { Intermediate users } \\
6-29 \text { days in } \\
\text { past } 30 \text {, } \\
\%(95 \% \mathrm{Cl})\end{array}$ & $\begin{array}{l}\text { Daily users } \\
\text { Every day in past 30, } \\
\%(95 \% \mathrm{Cl})\end{array}$ \\
\hline \multicolumn{5}{|l|}{ Goal oriented } \\
\hline To quit other tobacco products & $39.8(35.6$ to 43.9$)$ & $41.2(33.5$ to 48.9$)$ & 76.6 (65.7 to 87.5$)$ & 86.3 (77.8 to 94.8$)$ \\
\hline To cut down on other tobacco products & 42.7 (38.5 to 46.9$)$ & $49.3(41.3$ to 57.3$)$ & 84.6 (75.0 to 94.2$)$ & 91.0 (84.7 to 97.2$)$ \\
\hline Since they might be less harmful than other tobacco products & 45.3 (41.0 to 49.6$)$ & $54.5(46.5$ to 62.5$)$ & 63.9 (50.6 to 77.2$)$ & 87.3 (76.9 to 97.7$)$ \\
\hline To use them in places where other tobacco is not allowed & $32.3(28.3$ to 36.3$)$ & 46.9 (38.9 to 54.8$)$ & $58.2(45.4$ to 70.9$)$ & $65.6(52.9$ to 78.3$)$ \\
\hline Since they are affordable & 21.4 (17.9 to 24.9$)$ & $36.3(28.5$ to 44.1$)$ & 65.5 (52.9 to 78.1$)$ & $74.5(62.9$ to 86.0$)$ \\
\hline \multicolumn{5}{|l|}{ Non-goal oriented } \\
\hline Owing to curiosity about e-cigarettes & 81.9 (78.6 to 85.2$)$ & $79.2(72.9$ to 85.5$)$ & 64.0 (51.6 to 76.3 ) & $51.8(38.2$ to 65.3$)$ \\
\hline Since they come in menthol flavour & $11.9(9.1$ to 14.7$)$ & $11.6(6.7$ to 16.5$)$ & $18.4(7.8$ to 29.1$)$ & $17.7(7.3$ to 28.0$)$ \\
\hline Since they come in other flavours & 25.0 (21.2 to 28.8$)$ & 50.4 (42.4 to 58.4$)$ & 41.9 (28.9 to 54.9$)$ & 55.5 (42.2 to 68.8$)$ \\
\hline
\end{tabular}

within each use frequency category that endorsed each reason were numerically similar, but are not reported because small sample sizes prevented reliable inference.

Overall estimates of e-cigarette current use prevalence among Minnesota adults varied considerably depending on which types of users were included in the count: $6 \%(5.2 \%$ to $6.6 \%)$ if all user types were included; $2.4 \%$ (2\% to $2.9 \%)$ if intermediate and daily users were included; $1.1 \%(0.8 \%$ to $1.4 \%)$ if only daily users were included (table 1 ).

\section{DISCUSSION}

Defining e-cigarette current use prevalence as any use in the past 30 days failed to differentiate a cluster of infrequent users at the low end of the distribution from other users. In addition to having a distinctly different behavioural profile in terms of use frequency, infrequent users were more likely to report curiosity as a reason for using e-cigarettes and less likely to report goal-oriented reasons, compared to intermediate or daily users. These results suggest that many infrequent users are experimenters, unlikely to continue their e-cigarette use over time. ${ }^{16}$ If that is the case, then measuring e-cigarette current use prevalence based on any use in the past 30 days may lead to an overestimate of regular users. That conclusion is reinforced by the finding that most individuals who had ever used e-cigarettes reported no use in the past 30 days.

The importance of considering the distribution of days used has also been previously documented. Zhu et al $^{13}$ examined a nationally representative sample of e-cigarette users and similarly found the proportion of daily users was lower among current smokers $(11.5 \%)$ than recent former smokers (45.7\%). Other studies that have separated users at the top of the distribution from others have used the terms 'established users' ${ }^{20}$ and 'intensive users'. ${ }^{4}$ Clinically, a strict measure of use on 30 out of 30 days may have less utility than a broader category, because it may inadvertently exclude daily users who were temporarily abstinent for artificial reasons such as a broken device or serious illness.

A limitation of the current study is that we measured frequency of use in only a single, 30-day time window. We did not measure lifetime use of e-cigarettes; however, measures related to the quantity of use are problematic with e-cigarettes in light of the variety of types of cigarettes with varying quantities of nicotine and different associated use patterns. ${ }^{1}$ A more appropriate measure of use relevant to population health may be duration of use, measured by time since the individual first started e-cigarette use. Information on duration of use may be used in conjunction with numbers of days used in the past 30 days. Longitudinal data, such as the Population Assessment of Tobacco and Health study currently being conducted in the USA, will be particularly valuable for better understanding how e-cigarette use changes over time, how that use affects cigarette smoking, and how factors such as device type moderate these relationships. Another limitation is that we did not investigate demographic differences in this study, in order to keep this article focused on the primary methodological research question of operationalising prevalence. However, specific subpopulations may differ not only in overall prevalence, but also in the shape of their distributions, and more fine-grained questions about their cigarette and e-cigarette use may be necessary to accurately understand their behaviour. Finally, this study did not consider alternative measures of e-cigarette use such as number of times used per day or concentration of nicotine.

The 2012-2013 National Adult Tobacco Survey asked respondents, "Do you now use e-cigarettes every day, some days, rarely, or not at all?" The researchers included 'rarely' in the set of response options based on cognitive testing that suggested some participants felt neither 'some days' nor 'not at all' accurately described their use. Agaku et $a l^{14}$ report two separate estimates of e-cigarette current use prevalence: an estimate of $1.9 \%$ based only on responses of 'everyday' and 'some days', and another estimate of $4.2 \%$ that also included responses of 'rarely'. Our study investigated a different population (Minnesota vs USA) at a different time period (2014 vs 20122013). However, it is notable that the more restrictive definition in our study (use on more than 5 days; $2.4 \%$ ) yielded an estimate $60.0 \%$ smaller than the less restrictive definition (any use in the past 30 days; $6.0 \%$ ); the more restrictive definition reported by Agaku et $a l^{14}$ yielded an estimate that was similarly $54.8 \%$ smaller than their less restrictive definition. While further research allowing more direct comparison is needed, the similarity in magnitude between our study and Agaku et al. is at least suggestive that providing respondents with the 'rarely' option may be an important and effective method for differentiating experimenters from other users.

These results are consistent with an interpretation that many people are trying e-cigarettes, but few continue use. Furthermore, based on the sparsity in the centre of the distributions, the transition from infrequent to daily user appears to be fairly rapid for those who make it. If the transition were gradual, we would expect to see more individuals along the continuum at the level of intermediate users, which is not the 
case. There is evidence that some respondents' answers to the number of days question were influenced by a preference for round numbers, for example, local peaks are observed at 5,10 , 15,20 and 25 days. While the number of respondents at these local peaks in the centre of the distribution is too small to have substantially affected our conclusions, the preference for 5 may suggest that the distinct cluster of infrequent users observed in this self-report data may be less distinct in actual practice.

Previous research suggests that learning to obtain a 'satisfying' nicotine hit from current e-cigarette devices takes practice, as well as knowledge of nicotine concentrations and devices. ${ }^{24}$ One possibility is that regular users who are trying to quit cigarettes persist as infrequent users until they either learn to use e-cigarettes to effectively manage their nicotine addiction, at which point they quickly make the transition to daily users, or until they decide e-cigarettes are not for them and abandon the effort. If the current generation of ecigarettes were a more perfect substitute for nicotine delivery we would expect more daily use, a prediction supported by findings that established users tend to use different devices than the broader population of ever users. ${ }^{20}$ Frequency of use profiles may differ considerably based on device type; for example, daily users have a strong preference for refillable 'tank' devices. ${ }^{25}$ Survey items specific to particular device types and products will be useful for advancing research regarding how each tends to used, and for further differentiating types of users. At the same time, a consistent measure of use frequency across studies and devices will further our understanding of the relationship between device type and behaviour, and provide an efficient measure for public health surveillance.

\section{CONCLUSIONS}

These results suggest that defining e-cigarette current use as any reported use in the past 30 days captures a heterogeneous group of users at best and risks substantially overestimating its prevalence. Defining current use as 'more than 5 days out of the past $30^{\prime}$ may be a more accurate measure if the research goal is to estimate the proportion of a population that will persist in using e-cigarettes, but further research is necessary. Regardless of whether that definition or some other is adopted as a standard, greater consistency across studies is needed to better understand how the advent of e-cigarettes is (or is not) changing the landscape of tobacco use.

\section{What this paper adds}

- Electronic cigarette use has rapidly increased in recent years.

- Many tobacco control researchers are working to understand the extent of use and the implications.

- Unlike combustible cigarette smoking, a uniform definition for 'current use' of electronic cigarettes does not exist.

- That lack of standardisation presents a challenge to cross-study comparison, slowing progress.

- We found that defining prevalence as 'any 30-day use' included many users who were motivated by curiosity and were unlikely to continue use.

- This study identifies a methodological barrier to effective tobacco control policy regarding electronic cigarettes and uses a data-driven approach to propose a solution.
Twitter Follow Raymond Boyle at @Raymond_Boyle

Acknowledgements The authors are grateful to Ann St Claire, Ann Kinney, Pete Rode and the Westat team for collection of MATS. The authors wish to thank Mike Fiore, Bruce Christiansen, and Andrea Mowery for feedback on the manuscript.

Contributors RGB led design of the population survey and data collection. Discussions among all three authors shaped the conception of this article. MSA and RGB led the analysis and wrote the initial drafts. All three authors contributed to refinement of the final article language.

Funding This research was funded by ClearWay Minnesota, an independent non-profit organisation.

Competing interests None declared.

Ethics approval Minnesota Department of Health Institutional Review Board.

Provenance and peer review Not commissioned; externally peer reviewed.

Data sharing statement This manuscript draws on the 2014 Minnesota Adult Tobacco Survey (MATS). After planned analyses have been conducted and written up for submission, all data will be made publicly available to researchers on request through the ClearWay Minnesota website. Previous versions of MATS are currently available through the ClearWay Minnesota website.

\section{REFERENCES}

1 Zhu SH, Sun JY, Bonnevie E, et al. Four hundred and sixty brands of e-cigarettes and counting: implications for product regulation. Tob Control 2014;23(Suppl 3): iii3-9.

2 Esterl M. 'Vaporizers' are the new draw in e-cigarettes. Wall Street Journal 2014

3 King BA, Alam S, Promoff $G$, et al. Awareness and ever-use of electronic cigarettes among U.S. adults, 2010-2011. Nicotine Tob Res 2013;15:1623-7.

4 Biener L, Hargraves JL. A longitudinal study of electronic cigarette use in a population-based sample of adult smokers: association with smoking cessation and motivation to quit. Nicotine Tob Res 2015;17:127-33.

5 King BA, Patel R, Nguyen $\mathrm{KH}$, et al. Trends in awareness and use of electronic cigarettes among U.S. adults, 2010-2013. Nicotine Tob Res 2015;17:219-27.

6 McMillen RC, Gottlieb MA, Shaefer RM, et al. Trends in electronic cigarette use among U.S. adults: use is increasing in both smokers and nonsmokers. Nicotine Tob Res 2014. Published Online First: 6 Nov 2014. doi:10.1093/ntr/ntu213

7 Sanburn J. Can electronic cigarettes challenge big tobacco? Time 8 January 2013.

8 Delnevo CD, Bauer UE. Monitoring the tobacco use epidemic III: the host: data sources and methodological challenges. Prev Med 2009;48(1 Suppl):S16-23.

9 Bondy SJ, Victor JC, Diemert LM. Origin and use of the 100 cigarette criterion in tobacco surveys. Tob Control 2009;18:317-23.

10 U.S. Department of Health and Human Services. The Health Consequences of Smoking: 50 Years of Progress. A Report of the Surgeon General. Atlanta, GA: U. S. Department of Health and Human Services, Centers for Disease Control and Prevention, National Center for Chronic Disease Prevention and Health Promotion, Office on Smoking and Health, 2014.

11 Pulvers K, Hayes RB, Scheuermann TS, et al. Tobacco use, quitting behavior, and health characteristics among current electronic cigarette users in a national tri-ethnic adult stable smoker sample. Nicotine Tob Res Published Online Frst: 10 Nov 2014. doi:10.1093/ntr/ntu241

12 Christensen T, Welsh E, Faseru B. Profile of e-cigarette use and its relationship with cigarette quit attempts and abstinence in Kansas adults. Prev Med 2014;69C:90-4

13 Zhu SH, Gamst A, Lee M, et al. The use and perception of electronic cigarettes and snus among the U.S. population. PLOS ONE 2013;8:e79332.

14 Agaku IT, King BA, Husten CG, et al. Tobacco product use among adults-United States, 2012-2013. MMWR Morb Mortal Wkly Rep 2014;63:542-7.

15 Schmidt L, Reidmohr A, Harwell TS, et al. Prevalence and reasons for initiating use of electronic cigarettes among adults in Montana, 2013. Prev Chronic Dis 2014;11: E204.

16 Pepper JK, Ribisl KM, Emery SL, et al. Reasons for starting and stopping electronic cigarette use. Int J Environ Res Public Health 2014;11:10345-61.

17 Dockrell M, Morrison R, Bauld L, et al. E-cigarettes: prevalence and attitudes in Great Britain. Nicotine Tob Res 2013;15:1737-44

18 Gravely S, Fong GT, Cummings KM, et al. Awareness, trial, and current use of electronic cigarettes in 10 countries: findings from the ITC project. Int J Environ Res Public Health 2014;11:11691-704.

19 Gallus S, Lugo A, Pacifici R, et al. E-cigarette awareness, use, and harm perceptions in Italy: a national representative survey. Nicotine Tob Res 2014;16: 1541-8.

20 Giovenco DP, Lewis MJ, Delnevo CD. Factors associated with e-cigarette use: a national population survey of current and former smokers. Am J Prev Med 2014:47:476-80 
21 Delnevo CD, Lewis MJ, Kaufman I, et al. Defining cigarette smoking status in young adults: a comparison of adolescent vs adult measures. Am J Health Behav 2004;28:374-80.

22 Centers for Disease Control and Prevention (CDC). Smoking Status Recodes. 2009 http://www.cdc.gov/nchs/nhis/tobacco/tobacco_recodes.htm (accessed 16 Dec 2014) (cited 16 December 2014). http://www.cdc.gov/nchs/nhis/tobacco/tobacco_recodes.htm
23 AAPOR. Standard Definitions Final Dispositions of Case Codes and Outcome Rates for Surveys. 2011 (accessed Jan 2015).

24 McQueen A, Tower S, Sumner W. Interviews with "vapers": implications for future research with electronic cigarettes. Nicotine Tob Res 2011;13:860-7.

25 Foulds J, Veldheer S, Berg A. Electronic cigarettes (e-cigs): views of aficionados and clinical/public health perspectives. Int J Clin Pract 2011;65:1037-42. 